$$
\text { "tmcs-dlab" — 2011/11/20 - 19:35 — page } 225 \text { - \#1 }
$$

\title{
Arithmetic progressions of higher order
}

Vlastimil Dlab

Abstract. The aim of this article is to clarify the role of arithmetic progressions of higher order in the set of all progressions. It is important to perceive them as the pairs of progressions closely connected by simple relations of differential or cumulative progressions, i.e. by operations denoted in the text by $\Delta$ and $\nabla$. This duality affords in a natural way the concept of an alternating arithmetic progression that deserves further studies. All these progressions can be identified with polynomials and very special, explicitly described, recursive progressions. The results mentioned here point to a very close relationship among a series of mathematical objects and to the importance of combinatorial numbers; they are presented in a form accessible to the graduates of secondary schools.

Key words and phrases: arithmetic progressions, polynomials, recursive progressions.

ZDM Subject Classification: D40, D80, H20, K20, U30.

\section{Introduction}

The progression

$$
a_{1}=1, a_{2}=3, a_{3}=8, a_{4}=20, a_{5}=43, a_{6}=81, a_{7}=138, \ldots
$$

is an arithmetic progression of the third order. It can be interpreted as a sequence of the values of the polynomial

$$
P(n)=\frac{1}{6}\left(4 n^{3}-15 n^{2}+29 n-12\right)
$$

Copyright (C) 2011 by University of Debrecen 


$$
\text { "tmcs-dlab" — 2011/11/20 — 19:35 — page } 226 \text { — \#2 }
$$

at integral points $n=1,2, \ldots$, or as a linear differential equation (a recursive sequence) of the fourth order:

$a_{1}=1, a_{2}=3, a_{3}=8, a_{4}=20 \quad a \quad a_{n+4}=4 a_{n+3}-6 a_{n+2}+4 a_{n+1}-a_{n}, n=1.2 \ldots$

In addition, the sum of the first $n$ members $\sum_{t=1}^{n} a_{t}=S(n)$ is the value of the polynomial

$$
S(n)=\frac{1}{6}\left(n^{4}-3 n^{3}+8 n^{2}\right) .
$$

This particular example illustrates the theory of the arithmetic progressions of higher order that is the subject of this article. Its purpose is to bring back to life one of the very interesting concepts of elementary mathematics that used to be an integral part of the secondary school curricula. This topic provides an opportunity to experiment and play with simple (but not entirely simple) relations among numbers and contributes to a deeper understanding of elementary mathematics and arithmetics. It provides an opportunity for the teachers to excite interests in mathematics. The concluding remarks offer, in particular, a lot of possibilities, both for teachers and students, to experiment and keep finding new relations among concepts that could otherwise seem dull and tedious.

In the course of writing this article, I have realized that in order to reach a full understanding of the arithmetic progressions, it is necessary to study them within a wider family of general progressions. This explains those few remarks included in the introductory section.

A section on the arithmetic series of higher order can be found in Weber's textbook [2] on pp. 50-53. Euler used in his Algebra [1], Section III, Chapter $\mathrm{V}$, the term "polygonal" numbers. In fact, these progressions of numbers were already considered as very interesting by Pierre de Fermat in his notes on Diophantus.

\section{General progressions}

Let $\Pi$ be the set of all progressions a of real numbers. ${ }^{1}$ If $\mathbf{a}=\left(a_{1}, a_{2}, \ldots\right.$, $\left.a_{s}, \ldots\right)=\left(a_{s} \mid 1 \leq s\right)$, let us write $\Delta_{1, s}=a_{s}$, and define recursively the (infinite square) matrix $\mathbf{M}(\mathbf{a})=\left(\Delta_{r, s}\right)_{1 \leq r, s}$, where

$$
\Delta_{r, s}=\Delta_{r-1, s+1}-\Delta_{r-1, s} \text { for all } 1<r \text { and } 1 \leq s .
$$

${ }^{1}$ All statements hold for progressions whose members are elements of an arbitrary infinite integral domain. However, in this article we shall concentrate mainly on progressions of integers. 
Denote the sequence $\left(\Delta_{r, 1} \mid 1 \leq r\right)$ by $\mathbf{b}$. Thus $\mathbf{b}=\left(b_{r} \mid 1 \leq r\right)$ with $b_{r}=\Delta_{r, 1}$; let us remark that $b_{1}=a_{1}$. Hence $\mathbf{a}$ is the first row and $\mathbf{b}$ the first column of the matrix $\mathbf{M}(\mathbf{a})$. Here, it may be appropriate to note that the entries of $\mathbf{M}(\mathbf{a})$ are related to the so-called forward and backward differences of a function.

Using the relation (1) we can derive readily that

$$
\Delta_{r, s}=\Delta_{r+1, s-1}+\Delta_{r, s-1} \text { for all } 1 \leq r \text { and } 2 \leq s .
$$

The relations (1) direct us to subtract horizontally, i.e. the rows of the matrix $\mathbf{M}(\mathbf{a})$ represent the difference progressions (progressions of differences). The columns of the matrix $\mathbf{M}(\mathbf{a})$ represent in view of prescriptions (2) cumulative progressions (progressions of sums): we add vertically.

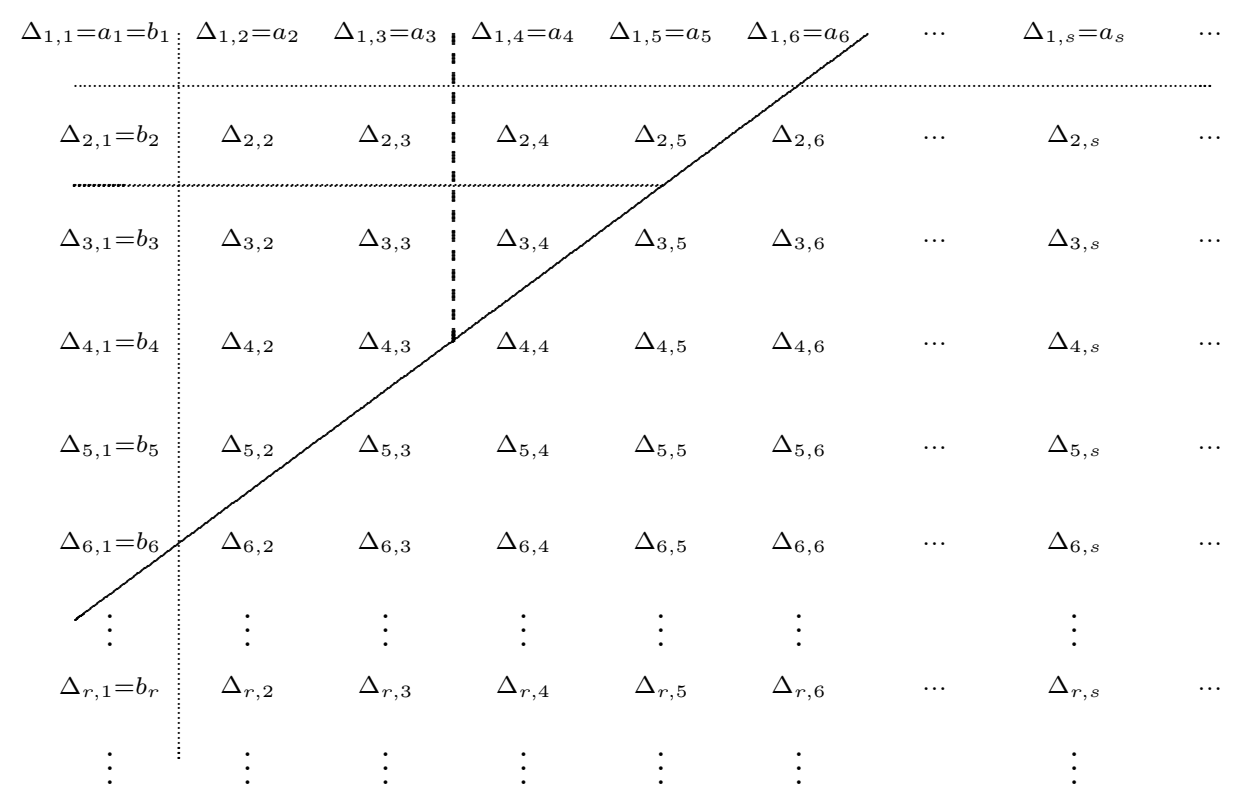

In this matrix, we can easily enumerate the relations among the marked entries. Using the relations (1), we get the equality

$$
\Delta_{3,4}=\Delta_{2,5}-\Delta_{2,4}=\Delta_{1,6}-\Delta_{1,5}-\left(\Delta_{1,5}-\Delta_{1,4}\right)=\Delta_{1,6}-2 \Delta_{1,5}+\Delta_{1,4},
$$

and using the relations (2)

$$
\begin{aligned}
\Delta_{3,4} & =\Delta_{4,3}+\Delta_{3,3}=\Delta_{5,2}+\Delta_{4,2}+\Delta_{4,2}+\Delta_{3,2}= \\
& =\Delta_{6,1}+\Delta_{5,1}+\Delta_{5,1}+\Delta_{4,1}+\Delta_{5,1}+\Delta_{4,1}+\Delta_{4,1}+\Delta_{3,1}= \\
& =\Delta_{6,1}+3 \Delta_{5,1}+3 \Delta_{4,1}+\Delta_{3,1} .
\end{aligned}
$$




$$
\text { "tmcs-dlab" — 2011/11/20 — 19:35 — page } 228 \text { — \#4 }
$$

These equalities are particular instances of the following important statement. These are formulae that express the element at the position $r, s$ by means of the elements of the first row, or the first column.

TheOREM 1. (a) For all $1 \leq r, s$,

$$
\Delta_{r, s}=\sum_{k=0}^{r-1}(-1)^{k}\left(\begin{array}{c}
r-1 \\
k
\end{array}\right) \Delta_{1, r+s-1-k}
$$

and thus

$$
b_{r}=\sum_{k=1}^{r}(-1)^{r-k}\left(\begin{array}{l}
r-1 \\
k-1
\end{array}\right) a_{k}
$$

(b) For all $1 \leq r, s$,

$$
\Delta_{r, s}=\sum_{k=0}^{s-1}\left(\begin{array}{c}
s-1 \\
k
\end{array}\right) \Delta_{r+s-1-k, 1},
$$

and thus

$$
a_{s}=\sum_{k=1}^{s}\left(\begin{array}{l}
s-1 \\
k-1
\end{array}\right) b_{k}
$$

Proof. The equalities (4) and (6) are simple consequences of the equalities (3) and (5); an interchange of order of the summands provides the result. They show the way to express the elements of the first row of the matrix $\mathbf{M}(\mathbf{a})$ by means of the elements of the first column and vice versa.

Since the proof of the equation (3) is similar to that of equation (5), we are going to prove (5) alone, leaving the proof of the relation (3) to the reader.

We prove the equality (5) by induction on $n=r+s$. The formula is trivial for $n=2$. Let us assume its validity for all $\Delta_{i, j}$, where $i+j<n$. For $r+s=n$, let us write $\Delta_{n-s, s}$. In this case, (5) is again trivial for $s=1$. Now we proceed by induction on $s$ : Assuming that (5) holds for $s$ we will show that (5) holds for $s+1$. Making use of the relation (2) and the induction hypothesis, we get

$$
\begin{aligned}
\Delta_{n-(s+1), s+1} & =\Delta_{n-s, s}+\Delta_{n-(s+1), s}= \\
& =\sum_{k=0}^{s-1}\left(\begin{array}{c}
s-1 \\
k
\end{array}\right) \Delta_{n-1-k, 1}+\sum_{k=1}^{s}\left(\begin{array}{c}
s-1 \\
k-1
\end{array}\right) \Delta_{n-1-k, 1}= \\
& =\Delta_{n-1,1}+\sum_{k=1}^{s-1}\left(\begin{array}{l}
s \\
k
\end{array}\right) \Delta_{n-1-k, 1}+\Delta_{n-1-s, 1}=\sum_{k=0}^{s}\left(\begin{array}{l}
s \\
k
\end{array}\right) \Delta_{n-1-k, 1},
\end{aligned}
$$

as required. 
Let us also formulate a simple relation for the sequence $\mathbf{s}=\left(s_{n}=\right.$ $\left.\sum_{k=1}^{n} a_{k} \mid 1 \leq n\right)$ of the partial sums of the progression $\mathbf{a}$.

TheOREM 2. For all $1 \leq n$,

$$
s_{n}=\sum_{s=1}^{n} a_{s}=\sum_{k=1}^{n}\left(\begin{array}{l}
n \\
k
\end{array}\right) b_{k} .
$$

ProOF. The equality (7) follows immediately by making use of the relation (6) and by interchanging the summands:

$$
s_{n}=\sum_{s=1}^{n} a_{s}=\sum_{s=1}^{n}\left(\sum_{k=1}^{s}\left(\begin{array}{l}
s-1 \\
k-1
\end{array}\right) b_{k}\right)=\sum_{k=1}^{n}\left(\sum_{s=k}^{n}\left(\begin{array}{l}
s-1 \\
k-1
\end{array}\right)\right) b_{k}=\sum_{k=1}^{n}\left(\begin{array}{l}
n \\
k
\end{array}\right) b_{k} .
$$

Here, we have used only the well-known sum

$$
\left(\begin{array}{l}
k-1 \\
k-1
\end{array}\right)+\left(\begin{array}{c}
k \\
k-1
\end{array}\right)+\left(\begin{array}{l}
k+1 \\
k-1
\end{array}\right)+\cdots+\left(\begin{array}{l}
n-1 \\
k-1
\end{array}\right)=\left(\begin{array}{l}
n \\
k
\end{array}\right)
$$

Now, we are going to define two mappings $\Delta: \Pi \rightarrow \Pi$ and $\nabla: \Pi \rightarrow \Pi$, i.e. two transformations of the set of all progressions of real numbers:

If $\mathbf{a}=\left(a_{s} \mid 1 \leq s\right)$ and $\mathbf{b}=\left(b_{r} \mid 1 \leq r\right)$, then

$$
\begin{aligned}
& \boldsymbol{\Delta}(\mathbf{a})=\mathbf{b}, \text { where } b_{r} \text { are given by the relation }(4) \text { and } \\
& \nabla(\mathbf{b})=\mathbf{a} \text {, where } a_{r} \text { are given by the relation }(6) .
\end{aligned}
$$

TheOREM 3. The mappings $\boldsymbol{\Delta}$ and $\nabla$ are mutually inverse (and thus bijective) mappings of the set $\Pi$, i.e.

$$
\Delta \nabla=\nabla \Delta=\mathbf{I}_{\Pi}
$$

where $\mathbf{I}_{\Pi}$ is the identity mapping of the set $\Pi$.

ProOF. Let us prove that $\boldsymbol{\Delta} \nabla=\mathbf{I}_{\Pi}$. The proof that $\nabla \boldsymbol{\Delta}=\mathbf{I}_{\Pi}$ is similar. It follows from the above definitions that for $\boldsymbol{\Delta} \nabla(\mathbf{b})=(\mathbf{c})=\left(c_{t} \mid 1 \leq t\right)$ one gets

$$
\begin{aligned}
c_{t} & =\sum_{k=1}^{t}(-1)^{t-k}\left(\begin{array}{l}
t-1 \\
k-1
\end{array}\right) \sum_{l=1}^{k}\left(\begin{array}{c}
k-1 \\
l-1
\end{array}\right) b_{l}=\sum_{l=1}^{t} \sum_{k=l}^{t}(-1)^{t-k}\left(\begin{array}{c}
t-1 \\
k-1
\end{array}\right)\left(\begin{array}{c}
k-1 \\
l-1
\end{array}\right) b_{l}= \\
& =\sum_{l=1}^{t}(-1)^{t}\left(\begin{array}{c}
t-1 \\
t-l
\end{array}\right)\left(\sum_{k=l}^{t}(-1)^{k}\left(\begin{array}{c}
t-l \\
k-l
\end{array}\right)\right) b_{l},
\end{aligned}
$$




$$
\text { "tmcs-dlab" — 2011/11/20 - 19:35 — page } 230 \text { — \#6 }
$$

for

$$
\left(\begin{array}{c}
t-1 \\
k-1
\end{array}\right)\left(\begin{array}{c}
k-1 \\
l-1
\end{array}\right)=\left(\begin{array}{c}
t-1 \\
t-l
\end{array}\right)\left(\begin{array}{l}
t-l \\
k-l
\end{array}\right)
$$

Moreover,

$$
\sum_{k=l}^{t}(-1)^{k}\left(\begin{array}{c}
t-l \\
k-l
\end{array}\right)=\sum_{p=0}^{t-l}(-1)^{p+l}\left(\begin{array}{c}
t-l \\
p
\end{array}\right)
$$

this is equal to 0 for $l<t$ and $(-1)^{l}$ for $l=t$. Therefore $c_{t}=(-1)^{2 t}\left(\begin{array}{c}t-1 \\ 0\end{array}\right) b_{t}=b_{t}$, and hence $\Delta \nabla(\mathbf{b})=\mathbf{b}$.

REMARK 1. Of course, using the matrix $\mathbf{M}(\mathbf{a})$ (i.e. using the relations (1) and (2)) the proof of Theorem 3. follows right away. As we have seen, the important part of the proof was the equality

$$
\sum_{k=s}^{r}(-1)^{k}\left(\begin{array}{l}
r \\
k
\end{array}\right)\left(\begin{array}{l}
k \\
s
\end{array}\right)=0 \text { for all } s<r .
$$

Now, it is only appropriate to illustrate some use of the mappings $\boldsymbol{\Delta}$ and $\nabla$, i.e. show the importance of the relations (4) and (6). By doing so, i.e. by choosing a suitable progression a and application of the relation (6), we get interesting expressions of the members $a_{s}$. For instance, the choice of the popular progression of Fibonacci $\mathbf{F}=\left(F_{s} \mid 1 \leq s\right)$, satisfying $F_{1}=F_{2}=1$ and $F_{s+2}=F_{s+1}+F_{s}$, results in the alternating Fibonacci progression $\boldsymbol{\Delta}(\mathbf{F})$, and from there, by the formula (6), to the following expression for the $n$-th Fibonacci number:

$$
F_{n}=1+\sum_{k=2}^{n-1}(-1)^{k}\left(\begin{array}{c}
n-1 \\
k
\end{array}\right) F_{k-1} .
$$

It is also easy to see that the $\boldsymbol{\Delta}$-image of a geometric progression is again a geometric progression. In other words, the subset of all geometric progressions is with respect to the mapping $\boldsymbol{\Delta}$ (and thus also with respect to the mapping $\nabla$ ) an invariant subset. Let us denote by $\mathbf{g}_{(a, q)}$ the geometric progression whose first term is $a$ and whose common ratio is $q$, and formulate this fact explicitly.

TheOREM 4. $\boldsymbol{\Delta}\left(\mathbf{g}_{(a, q)}\right)=\mathbf{g}_{(a, q-1)}$, and thus $\nabla\left(\mathbf{g}_{(a, q)}\right)=\mathbf{g}_{(a, q+1)}$. This means that the mappings $\boldsymbol{\Delta}$ and $\nabla$ induce on the subset $\Pi_{(\mathbf{q})} \subset \Pi$ of all geometric progressions bijective mappings.

A similar theorem can be formulated for the subset $\Pi_{(\mathbf{p}, \mathbf{q})} \subset \Pi$ of all recursive sequences $\mathbf{a}=\left(a_{1}, a_{2} ; p, q\right)$, which satisfy $a_{n+2}=p a_{n+1}+q a_{n}$ for all $1 \leq n$ (to recall the concept, see Definition 2 below in the text). 
ThEOREM 5. The mappings $\boldsymbol{\Delta}$ and $\nabla$ induce a bijective transformation of the subset $\Pi_{(\mathbf{p}, \mathbf{q})}$ :

$$
\begin{aligned}
& \Delta\left(a_{1}, a_{2} ; p, q\right)=\left(a_{1}, a_{2}-a_{1} ; p-2, p+q-1\right), \text { and thus } \\
& \nabla\left(a_{1}, a_{2} ; p, q\right)=\left(a_{1}, a_{1}+a_{2} ; p+2, q-p-1\right) .
\end{aligned}
$$

Now, denote by $\mathbf{a}_{(a, d)}$ the arithmetic progression whose first term is $a$ and whose common difference is $d$. We can see immediately that $\mathbf{b}=\boldsymbol{\Delta}\left(\mathbf{a}_{(a, a)}\right)$ satisfies $b_{1}=a, b_{2}=d$ and $b_{r}=0$ for all $r \geq 3$. This is the most simple example of the main subject of this article.

In this connection, let us introduce a less trivial example. Consider the progression a satisfying $a_{s}=\left(\begin{array}{c}n+s-1 \\ k\end{array}\right)$. Write $\mathbf{b}=\boldsymbol{\Delta}(\mathbf{a})$. Then $b_{r}=\left(\begin{array}{c}n \\ k-r+1\end{array}\right)$ for $r \leq k+1$ and $b_{r}=0$ for $r \geq k+2$. Using the relation (6), we get

$$
\begin{array}{ll}
a_{s}=\sum_{l=0}^{s-1}\left(\begin{array}{c}
s-1 \\
l
\end{array}\right)\left(\begin{array}{c}
n \\
k-l
\end{array}\right) \quad \text { for } s \leq k+1 \text { and } \\
a_{s}=\sum_{l=0}^{k}\left(\begin{array}{c}
s-1 \\
l
\end{array}\right)\left(\begin{array}{c}
n \\
k-l
\end{array}\right) \quad \text { for } s>k+1 .
\end{array}
$$

For instance, this means for $k=2$ that for $s>3$,

$$
a_{s}=\left(\begin{array}{c}
s-1 \\
0
\end{array}\right)\left(\begin{array}{l}
n \\
2
\end{array}\right)+\left(\begin{array}{c}
s-1 \\
1
\end{array}\right)\left(\begin{array}{l}
n \\
1
\end{array}\right)+\left(\begin{array}{c}
s-1 \\
2
\end{array}\right)\left(\begin{array}{l}
n \\
0
\end{array}\right),
$$

and, as we shall see in the next section, $a_{s}$ is the value of the polynomial

$$
P_{\mathbf{a}}(x)=\frac{1}{2}\left[x^{2}+(2 n-3) x+n^{2}-3 n+2\right]
$$

for $x=s$

\section{Arithmetic progressions of higher order}

Finally, we are getting to the very subject of this article. In the preceeding section, we have introduced an example of a progression a such that all members of the progression $\mathbf{b}=\boldsymbol{\Delta}(\mathbf{a})$ were, from a certain index $n_{0}$, zero, i.e. $b_{r}=0$ for all $r \geq n_{0}$. In view of the formula (2), this property is equivalent to $\Delta_{n_{0}, s}=0$ for all $1 \leq s$, i.e. with the fact that the $n_{0}$-th row (and all the following ones) of the matrix $\mathbf{M}(\mathbf{a})$ are zero. This calls for the following definition. 


$$
\text { "tmcs-dlab" — 2011/11/20 - 19:35 — page } 232 \text { — \#8 }
$$

Definition 1. The progression $\mathbf{a}=\left(a_{s} \mid 1 \leq s\right)$ is said to be an arithmetic progression of higher order, if there exists $r$ such that $\Delta_{r, s}=0$ for all $1 \leq s$. The least $d$ such that $\Delta_{d+2, s}=0$ for all $1 \leq s$ is called the order of this arithmetic progression.

REMARK 2. Thus, in this terminology, the non-stationary arithmetic progressions, as we know them from elementary algebra, are arithmetic progressions of order 1 . The arithmetic progressions that satisfy $a_{s}=c \neq 0$ for all $1 \leq s$, are the arithmetic progressions of order 0 , while the zero progression is an arithmetic progression of order -1 (or we may say that it does not have order).

The Fibonacci progression $\mathbf{F}=\left(F_{s} \mid 1 \leq s\right)$ mentioned earlier, is not an arithmetic progression. We can see easily that in the above scheme for this progression the entries satisfy

$$
\Delta_{1, s}=\Delta_{r, 2 r+s-2} \quad \text { for all } 1 \leq s, 1 \leq r
$$

Moreover, no geometric progression $\mathbf{g}_{(a, q)}$ with $a \neq 0$ and $q \neq 1$, is an arithmetic progression. On the other hand, the progression $\mathbf{p}_{\mathbf{k}}=\left(a_{s}=s^{k} \mid 1 \leq s\right)$ is an arithmetic progression of degree $k$ for an arbitrary natural number $k$.

REMARK 3. In keeping with the relation (2), a progression a is an arithmetic progression of order $d$ if and only if $\mathbf{b}=\boldsymbol{\Delta}(\mathbf{a})$ satisfies the condition

$$
b_{d+1} \neq 0 \text { a } b_{r}=0 \quad \text { for all } d+2 \leq r .
$$

Hence, arithmetic progressions of higher order are just those progressions a for which $\boldsymbol{\Delta}(\mathbf{a})$ has only a finite number of non-zero terms.

REMARK 4. In what follows, some equalities will contain symbols $\left(\begin{array}{l}x \\ k\end{array}\right)$. Therefore, let us recall that the definition of the combinatorial numbers (binomial coefficients) can easily be extended for arbitrary numbers $x$ (and natural numbers $k)$ :

$$
\left(\begin{array}{l}
x \\
k
\end{array}\right)=\frac{x(x-1)(x-2) \ldots(x-k+1)}{k !}
$$

For instance, $\left(\begin{array}{l}\pi \\ 2\end{array}\right)$ is simply the real number $\frac{\pi(\pi-1)}{2}$, viz. the value of the quadratic polynomial (parabola) $y=\frac{1}{2}\left(x-\frac{1}{2}\right)^{2}-\frac{1}{8}$ at the point $x=\pi$. It may be worthy to recall at this point that our progressions do not have to be integral. 


$$
\text { "tmcs-dlab" — 2011/11/20 — 19:35 — page } 233 \text { — \#9 }
$$

Now, we are in position to formulate a few theorems, namely some consequences of Theorem 1.

TheOREM 6. To any arithmetic progression $\mathbf{a}=\left(a_{s} \mid 1 \leq s\right)$ of order $d$ there correspond a unique polynomial $V_{\mathbf{a}}(x)$ of degree $d$ such that $V_{\mathbf{a}}(s)=a_{s}$ and a unique polynomial $S_{\mathbf{a}}(x)$ of degree $d+1$ such that its absolute term is zero and $S_{\mathbf{a}}(n)=\sum_{s=1}^{n} a_{s}$.

Proof. Applying Theorem 1 and Theorem 2 (namely the equalities (6) and (7)) one can see easily that

$$
V_{\mathbf{a}}(x)=\sum_{k=1}^{d+1}\left(\begin{array}{l}
x-1 \\
k-1
\end{array}\right) b_{k}
$$

and

$$
S_{\mathbf{a}}(x)=\sum_{k=1}^{d+1}\left(\begin{array}{l}
x \\
k
\end{array}\right) b_{k}
$$

Equally easily one can foresee that every polynomial $P(x)=\sum_{n=0}^{d} A_{n} x^{n}$ is of the form $V_{\mathbf{a}}(x)$ for a suitably chosen progression $\mathbf{b}=\left(b_{r} \mid 1 \leq r \leq d+1\right)$. Let us record this fact explicitly in the following form.

TheOREM 7. Let $P(x)=\sum_{n=0}^{d} A_{n} x^{n}$ be an arbitrary polynomial of degree $d$ (i.e. $\left.A_{d} \neq 0\right)$. Let $M=\left(z_{m, r}\right)$ be a $(d+1) \times(d+1)$ be a matrix defined by means of the equalities

$$
z_{m, r}=\sum_{k=0}^{r-1}(-1)^{k}\left(\begin{array}{c}
r-1 \\
k
\end{array}\right)(r-k)^{m-1}
$$

Let us write $\mathbf{A}=\left(A_{0}, A_{1}, \ldots, A_{d}\right)$ and define $\mathbf{B}=\left(b_{1}, b_{2}, \ldots, b_{d+1}\right)$ by means of

$$
\mathbf{B}=\mathbf{A} M
$$

Then the progressions $\mathbf{a}=\left(a_{s}=P(s) \mid 1 \leq s\right)$ and $\mathbf{b}=\left(b_{r} \mid b_{r}=0\right.$ prod $\left.+2 \leq r\right)$ satisfy

$$
\mathbf{b}=\boldsymbol{\Delta}(\mathbf{a}), \text { and therefore also } \mathbf{a}=\nabla(\mathbf{b})
$$




$$
\text { "tmcs-dlab" — 2011/11/20 — 19:35 — page } 234 \text { — \#10 }
$$

Proof. The following proof is very simple. By (3) or (4),

$$
\begin{aligned}
b_{r} & =\sum_{k=0}^{r-1}(-1)^{k}\left(\begin{array}{c}
r-1 \\
k
\end{array}\right) b_{r-k}=\sum_{k=0}^{r-1}(-1)^{k}\left(\begin{array}{c}
r-1 \\
k
\end{array}\right) \sum_{t=0}^{n} A_{t}(r-k)^{t}= \\
& =\sum_{t=0}^{n}\left(\sum_{k=0}^{r-1}(-1)^{k}\left(\begin{array}{c}
r-1 \\
k
\end{array}\right)(r-k)^{t}\right) A_{t}=\sum_{t=0}^{n} A_{t} z_{t+1, r} .
\end{aligned}
$$

Corollary 1. There is a bijective correspondence between the polynomials and arithmetic progressions of higher order. To every polynomial $P(x)$ of degree $d$ there corresponds the arithmetic progression $\mathbf{a}_{P}=\left(a_{s}=P(s) \mid 1 \leq s\right)$ of order $d$. On the contrary, every arithmetic progression a of order $d$ there corresponds the polynomial (8) of degree $d$, where $b_{k}$ is the $k$-th member of the progression $\mathbf{b}=\Delta(\mathbf{a})$.

REMARK 5. Employing the formula (9), it is possible as above, to establish a one-to-one correspondence between the arithmetic progressions of order $d$ and the polynomials of degree $d+1$ with zero absolute term. It may be appropriate to present a general formulation of the above correspondence: Let I be an infinite integral domain. Then the correspondence of Corollary is an isomorphism between the additive group of all arithmetic progressions of higher order with terms from the domain $I$ and the additive group of all polynomials from the domain $I[x]$.

REMARK 6. Observe that the elements $z_{m, r}$ of the matrix $M$ in Theorem 7 . are, up to a multiple, Sterling numbers $S(m, r)$ of the second kind:

$$
z_{m, r}=(k-1) ! S(m, r) .
$$

Recall that $S(m, r)$ is the number of possibilities how to divide a set of $m$ elements into $r$ non-empty subsets. Hence, the form of the matrix $M$ is for $d=7$ as follows:

$$
M=\left(\begin{array}{cccccccc}
1 & 0 & 0 & 0 & 0 & 0 & 0 & 0 \\
1 & 1 & 0 & 0 & 0 & 0 & 0 & 0 \\
1 & 3 & 2 & 0 & 0 & 0 & 0 & 0 \\
1 & 7 & 12 & 6 & 0 & 0 & 0 & 0 \\
1 & 15 & 50 & 60 & 24 & 0 & 0 & 0 \\
1 & 31 & 180 & 390 & 360 & 120 & 0 & 0 \\
1 & 63 & 602 & 2100 & 3360 & 2520 & 720 & 0 \\
1 & 127 & 1932 & 10206 & 25200 & 31920 & 20160 & 5040
\end{array}\right)
$$




$$
\text { "tmcs-dlab" — 2011/11/20 — 19:35 — page 235 — \#11 }
$$

The following assertion indicates the fact that the set of all arithmetic progressions of higher order is very large.

THEOREM 8. An arbitrary sequence of $k$ numbers $\left(a_{s} \mid 1 \leq s \leq k\right)$ can be (uniquely) extended to an arithmetic progression $\mathbf{a}=\left(a_{s} \mid 1 \leq s\right)$ of order $d \leq k-1$.

Proof. The finite sequence $\left(a_{s} \mid 1 \leq s \leq k\right)$ determines uniquely all $\Delta_{r, s}$ for $r+s \geq k+1$, and thus $b_{r}=\Delta_{r, 1}$ for $1 \leq r \leq k$, in particular. Set $b_{r}=0$ for all remaining $r$, i.e. $k+1 \leq r$ and denote the resulting progression by $\mathbf{b}$. Then the progression $\mathbf{a}=\nabla(\mathbf{b})$ is the requested extension.

A full understanding of Theorem 8 (and Theorem 1) requires an introduction of the concept of a linear recursive sequence depending on a finite number of parameters.

Definition 2. A sequence $\mathbf{a}=\left(a_{s} \mid 1 \leq s\right)$ is said to be a linear recursive sequence of order $h$ (depending on $h$ parameters) if there are non-zero numbers $p_{1}, p_{2}, \ldots, p_{h}$ such that

$$
a_{n+h}=p_{1} a_{n+h-1}+p_{2} a_{n+h-2}+\cdots+p_{h} a_{n}=\sum_{t=1}^{h} p_{t} a_{n+h-t} \text { for all } 1 \leq n .
$$

Such a sequence will be denoted by $\mathbf{a}=\left(a_{1}, a_{2}, \ldots, a_{h} ; p_{1}, p_{2}, \ldots, p_{h}\right)$.

ThEOREM 9. The arithmetic progressions a of order $d$ are precisely the linear recursive sequences of order $d+1$ :

$$
\begin{gathered}
\mathbf{a}=\left(a_{1}, a_{2}, \ldots, a_{d+1} ; p_{1}, p_{2}, \ldots, p_{d+1}\right) \\
\text { where } p_{t}=(-1)^{t-1}\left(\begin{array}{c}
d+1 \\
t
\end{array}\right) \quad \text { for } 1 \leq t \leq d+1
\end{gathered}
$$

PROOF. The requirement

$$
a_{n}=\sum_{t=1}^{d+1}(-1)^{t-1}\left(\begin{array}{c}
d+1 \\
t
\end{array}\right) a_{n-t} \quad \text { for all } d+2 \leq n
$$

is just the equality (3) in Theorem 1 for $r=d+2$ and $s=n-d-1$. Indeed, by the assumption, $\Delta_{d+2, s}=0$ for all $1 \leq s$. 
It may be useful to emphasize the bijective correspondences derived above. The arithmetic progressions of order $d$ can be identified with the polynomials of degree $d$ and also with specific recursive sequences of order $d+1$. These identifications, that is the respective bijective mappings, are the essence of Theorem 6 and Theorem 9. Thus, for instance, the ordinary arithmetical progression (i.e. a progression of order 1$) \mathbf{a}_{(a, d)}$ is the recursive sequence $(a, a+d ; 2,-1)$ and the respective polynomial is $P_{\mathbf{a}}(x)=d x+(a-d)$. Similarly, the arithmetic progression of order 2 whose first three terms are $\left(a_{1}, a_{2}, a_{3}\right)$ is the recursive sequence $\left(a_{1}, a_{2}, a_{3} ; 3,-3,1\right)$ and the corresponding polynomial is $P_{\mathbf{a}}(x)=$ $\frac{1}{2}\left(a_{3}-2 a_{2}+a_{1}\right) x^{2}+\frac{1}{2}\left(-3 a_{3}+8 a_{2}-5 a_{1}\right) x+\left(a_{3}-3 a_{2}+3 a_{1}\right)$.

Let us use this opportunity to introduce a concept that is "dual", to the concept of an arithmetic progression, and that will merit further investigations. While the arithmetic progressions (of first order) a are characterized by the fact that the progression $\mathbf{b}=\Delta(\mathbf{a})=\left(b_{r} \mid 1 \leq r\right)$ satisfies $b_{r}=0$ for all $3 \leq r$, let us define progressions $\Delta(\mathbf{a})$ such that the progression $\mathbf{a}=\left(a_{s} \mid 1 \leq s\right)$ satisfies $a_{s}=0$ for all $3 \leq s$, and let us do it in a general form.

Definition 3. A progression $\mathbf{b}$ is said to be an alternating arithmetic progression of order $d$ if $\nabla(\mathbf{b})=\mathbf{a}=\left(a_{s} \mid 1 \leq s\right)$ satisfies $a_{s}=0$ for all $d+2 \leq s$ and $a_{d+1} \neq 0$.

A great deal of the above results on arithmetic progressions can be translated into the terminology of the alternating arithmetic progressions. However, one should be aware of the fact that some definite differences take place already for the progressions of order 1 . It is easy to derive for such progressions that

$$
b_{r}=(-1)^{r}\left[-b_{1}+(r-1)\left(b_{1}+b_{2}\right)\right]
$$

and that the sum of the first $n$ terms of such progressions is $s_{n}=t\left(b_{1}+b_{2}\right)$ for even $n=2 t$ and $s_{n}=b_{1}-(t-1)\left(b_{1}+b_{2}\right)$ for odd $n=2 t-1$. Let us add that these progressions are the recursive sequences $\left(b_{1}, b_{2} ;-2,-1\right)$.

\section{Illustrations}

A typical example of the arithmetic progressions of higher order are the progressions of the $k$-th powers of natural numbers $(k \geq 1)$. If we consider progressions over a number field (e.g. over real numbers) then the set of all arithmetic 


$$
\text { "tmcs-dlab" — 2011/11/20 — 19:35 — page } 237 \text { — \#13 }
$$

progressions form a vector space over that field and the subset of all progressions of the $k$-th powers of natural numbers forms a basis of that space. It is due to this fact that there is such a close relationship between these progressions and the polynomials!

Considering, for example, the sum $\sum_{s=1}^{n} s^{3}$, we get immediately from the scheme

$\begin{array}{lcccc}1 & 8 & 27 & 64 & 125 \\ 7 & 19 & 37 & 61 & \\ 12 & 18 & 24 & & \\ 6 & 6 & & & \\ 0 & & & & \end{array}$

and from the formula (7) the equality

$$
\sum_{s=1}^{n} s^{3}=\left(\begin{array}{l}
n \\
1
\end{array}\right)+7\left(\begin{array}{l}
n \\
2
\end{array}\right)+12\left(\begin{array}{l}
n \\
3
\end{array}\right)+6\left(\begin{array}{l}
n \\
4
\end{array}\right)=\frac{n^{2}(n+1)^{2}}{4}
$$

In a similar way, dealing with the progression

$$
1,32,243,1024,3125,7776,16807, \ldots
$$

we get successively $\Delta_{2,1}=31, \Delta_{3,1}=180, \Delta_{4,1}=390, \Delta_{5,1}=360, \Delta_{6,1}=120$ and $\Delta_{7,1}=0$, and hence

$$
\sum_{s=1}^{n} s^{5}=\left(\begin{array}{l}
n \\
1
\end{array}\right)+31\left(\begin{array}{l}
n \\
2
\end{array}\right)+180\left(\begin{array}{l}
n \\
3
\end{array}\right)+390\left(\begin{array}{l}
n \\
4
\end{array}\right)+360\left(\begin{array}{l}
n \\
5
\end{array}\right)+120\left(\begin{array}{l}
n \\
6
\end{array}\right) .
$$

If the progression $\mathbf{a}$ is defined by a polynomial $P_{\mathbf{a}}(x)$, then the value of the corresponding polynomial $S_{\mathbf{a}}(x)$ for $x=n$ yields the sum $\sum_{t=1}^{n} P_{\mathbf{a}}(t)$. So, for example, given the polynomial $P_{\mathbf{a}}(x)=a x^{2}+b x+c$, we can easily find that $\Delta_{1,1}=a+b+c, \Delta_{2,1}=3 a+b$ and $\Delta_{3,1}=2 a ;$ from here,

$$
S_{\mathbf{a}}(x)=\frac{1}{6}\left[2 a x^{3}+3(a+b) x^{2}+(a+3 b+6 c) x\right]=\int P_{\mathbf{a}}(x) d x+C_{\mathbf{a}}(x),
$$

with the correcting polynomial

$$
C_{\mathbf{a}}(x)=\frac{a}{2} x^{2}+\left(\frac{a}{6}+\frac{b}{2}\right) x
$$


If we choose $a=1, b=c=0$, we get a well-known formula

$$
\sum_{s=1}^{n} s^{2}=\frac{1}{3} n^{3}+\frac{1}{2} n^{2}+\frac{1}{6} n=\frac{n(n+1)(2 n+1)}{6} .
$$

Similarly, for the progression a determined by the polynomial $P_{\mathbf{a}}(x)=a x^{3}+$ $b x^{2}+c x+d$ we get that

$$
S_{\mathbf{a}}(x)=\int P_{\mathbf{a}}(X) d x+C_{\mathbf{a}}(x),
$$

where

$$
C_{\mathbf{a}}(x)=\frac{a}{2} x^{3}+\left(\frac{a}{4}+\frac{b}{2}\right) x^{2}+\left(\frac{b}{6}+\frac{c}{2}\right) x .
$$

Now, we get for $a=1, b=c=d=0$, the formula

$$
\sum_{s=1}^{n} s^{3}=\frac{1}{4} n^{4}+\frac{1}{2} n^{3}+\frac{1}{4} n^{2}=\frac{n^{2}(n+1)^{2}}{4} .
$$

Finally, we include also an illustration of Theorem 8. Let us determine the arithmetic progression $\mathbf{a}=\left(a_{s} \mid 1 \leq s\right)$ řádu $\leq 3$ such that

$$
a_{1}=5, a_{2}=-1, a_{3}=2, a_{4}=0 \text {. }
$$

Using our scheme

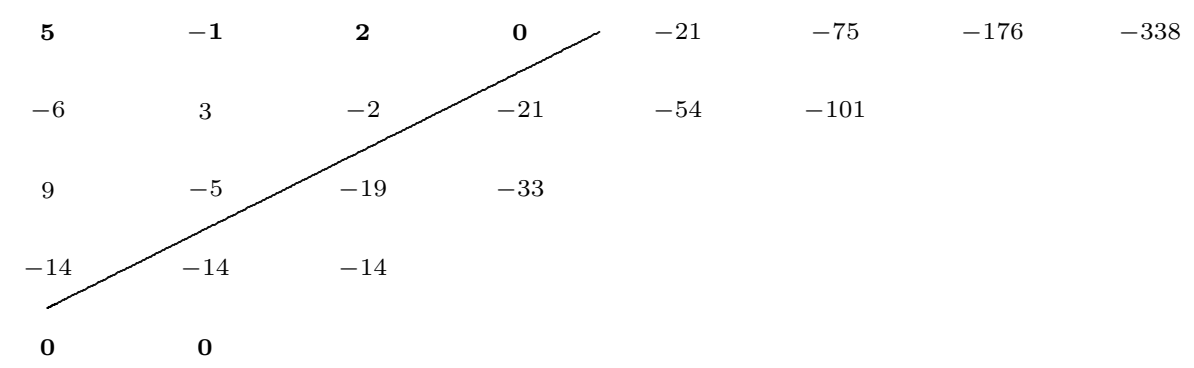

we get

$$
S_{\mathbf{a}}(x)=5\left(\begin{array}{l}
x \\
1
\end{array}\right)-6\left(\begin{array}{l}
x \\
2
\end{array}\right)+9\left(\begin{array}{l}
x \\
3
\end{array}\right)-14\left(\begin{array}{l}
x \\
4
\end{array}\right)=\frac{1}{12} x\left(174-167 x+60 x^{2}-7 x^{3}\right) .
$$

From here,

$$
P_{\mathbf{a}}(x)=a_{s}=S_{\mathbf{a}}(x)-S_{\mathbf{a}}(x-1)=-\frac{1}{6}\left(14 x^{3}-111 x^{2}+271 x-204\right),
$$




$$
\text { "tmcs-dlab" — 2011/11/20 — 19:35 — page } 239 \text { — \#15 }
$$

and thus $a_{5}=-21, a_{6}=-75, a_{7}=-176, a_{8}=-338, a_{9}=-575, \ldots$ Of course, the polynomial expressing the values $a_{s}=P_{\mathbf{a}}(s)$ can be obtained also making use of Theorem 1, or directly applying Legrange interpolation.

The reader may like to check for him/herself that the progression of the three terms $\sqrt{2},-10,0$ can be extended to an arithmetic progression of order 2 whose general term is $a_{s}=\frac{1}{2}\left[(20+\sqrt{2}) s^{2}-(80+5 \sqrt{2}) s+60+6 \sqrt{2}\right]$ and the sum of the first $n$ members $S_{\mathbf{a}}(n)=\frac{1}{6} n\left[(20+\sqrt{2}) n^{2}-(90+6 \sqrt{2}) n+70+11 \sqrt{2}\right]$.

\section{References}

[1] L. Euler, Elements of Algebra, (Translation of Vollstandige Anleitung zur Algebra), Springer-Verlag, 1984.

[2] H. Weber, Lehrbuch der Algebra, Vol.I, 3rd Edition, Chelsea Publ. Comp. New York.

VLASTIMIL DLAB

CARLETON UNIVERSITY

OTTAWA

CANADA

E-mail: vdlab@math.carleton.ca

(Received December, 2010) 